\title{
Change in the feedback stimulus on maintenance of behavior under a free operant avoidance (FOA) schedule ${ }^{1}$
}

\section{A. E. ROBERTS and H. M. B. HURWITZ, University of Tennessee, Knoxville, Tenn. 37916}

Response-feedback conditions (FS) were manipulated so that for three hooded rats trained under a free-operant avoidance schedule, a session consisted of FS, no-FS, $F S$; for other Ss, it was no-FS, FS, no-FS. The latter Ss responded at a higher rate during the initial no-FS period, and maintained a high rate during subsequent periods as well as during sessions. The former group indicated no change in response rates compared with initial training conditions.

Under the free-operant avoidance schedule (FOA), shocks are given at regular intervals. When a response occurs, shock is postponed for a fixed period. A number of investigators have employed a response-contingent stimulus to provide feedback to an organism. The feedback stimulus has been studied as an independent variable affecting FOA acquisition (Bolles \& Popp, 1964; Leaf, 1966; Bolles \& Grossen, 1969 ), or has simply been used without any evaluation of its effect (Bolles \& Warren, 1965; Leaf, 1965; Sandler, Davidson, \& Holzschuh, 1966). No data is available about the effect of a feedback stimulus (FS)-or changes in FS-upon maintenance of FOA behavior after initial training has been completed. The purpose of this experiment was to assess the effects produced by a change in the feedback stimulus conditions after extensive training had been given. The focus was not upon the role of FS in acquisition, but upon maintenance of FOA behavior in trained Ss, as measured by changes in the rate of response and frequency of shocks.

\section{METHOD}

Six female hooded rats were used who had previously been run for 702 -h sessions in an experiment dealing with conditioned suppression of FOA responding. PROCEDURE

Shocks were programmed to occur at 5-sec intervals unless a barpress occurred; then shock was postponed for 20 sec. After completion of the earlier experiment, the $S s$ were retrained until a stable baseline of response was established. Houselights were turned on at the beginning of the session and, coincident with a barpress, were momentarily extinguished. This change in illumination served as the feedback stimulus (FS).

Two feedback conditions were studied, with three Ss for each of two conditions. The three $S s$ in each condition were run under a different shock intensity: $.8 \mathrm{~mA}$ $1.4 \mathrm{~mA}, 2.0 \mathrm{~mA}$ of $0.1-\mathrm{sec}$ duration. Two arrangements of feedback conditions were studied, $A B A_{1}$ and $B A B_{1}$. Each session was divided into three $30-\mathrm{min}$ periods, with each period having an A or B contingency. A refers to a barpress resulting in a $0.1-\mathrm{sec}$ offset of houselights. This was also the condition during original training sessions. $B$ refers to a barpress that did not result in a change in illumination. Upon completion of each of the periods, response and shock measures were recorded from counters. Four daily sessions were run for each $S$ in Condition ABA or BAB.

\section{RESULTS}

Figure 1 presents mean responses per minute for all four daily sessions for each $S$ over the three $30-\mathrm{min}$ periods. The upper panel presents data for Condition $\mathrm{ABA}_{1}$; the lower panel presents data for Condition $\mathrm{BAB}_{1}$. Response rates during the last session before the experimental procedure was introduced are indicated by $\mathbf{T}$.

Ss under Condition $\mathrm{ABA}_{1}$ indicated little systematic changes in response rate as a result of changes in FS condition. The rates were the same as those observed under $T$.

Ss under Condition $\mathrm{BAB}_{1}$ showed marked changes in response rates. The first 30 -min period (B) that did not result in change of illumination led to a high rate of responding on each of the 4 days, compared both to T, the last training session before introduction of this procedure, and to the subsequent period $A$, during which the conditions were the same as in training. Subsequent return to the final $30-\mathrm{min}$ B period did not result in a return to such high rates of responding, but rates remained substantially higher than recorded during training $(T)$ sessions and during the ABA conditions.

Figure 2 presents mean shocks per minute for all four daily sessions for each $S$ over the three 30 -min periods. The upper panel presents shock rates for Condition $A B A_{1}$, the lower panel presents shock rates for Condition $\mathrm{BAB}_{1}$. Shock rates obtained during the last session before the experimental procedure was introduced are indicated by $\mathrm{T}$. Increases in response rates observed under Condition $\mathrm{BAB}_{1}$ were reflected in reductions in shock rates when compared to $T$. The reciprocal relationship between response rate and shock rate can be seen in the data from $\mathrm{S} 3$, Condition $\mathrm{BAB}_{1}$. An increase in response rate during Condition $B$ resulted in a reduction in shock rate; the reduction in rate of responding under Conditions $A$ and $B_{1}$ resulted in comparable increases in shock rates. No systematic effects were attributable to different shock intensities. DISCUSSION

It has been suggested that, with extensive training, a $S$ relies upon fewer external and internal stimuli in the maintenance of a response pattern (Adams, 1968). From this point of view, the withdrawal of response-produced stimuli (FS) should not affect the rate of response. Hence, the transition from training condition ( $T$ ), when feedback stimulus was available, to Condition B, where no specific feedback occurred, should not have resulted in any marked behavioral change. A different prediction may be derived from the view that changes in stimulus conditions-whether external or
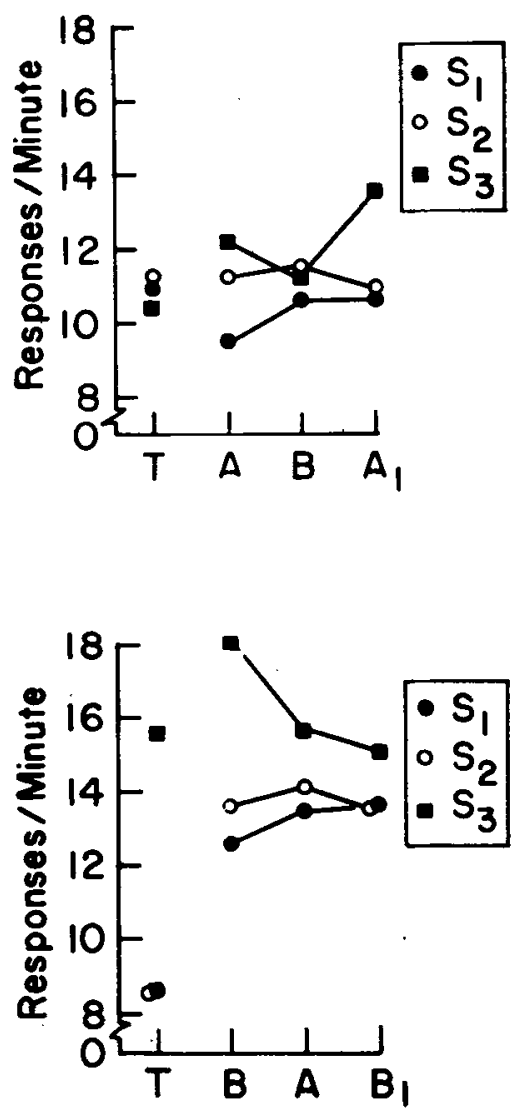

Fig. 1. Response rates for the last training session ( $T$ ), and mean response per minute over four sessions over successive 30 -min periods. A, feedback condition; $B$, feedback removed. 

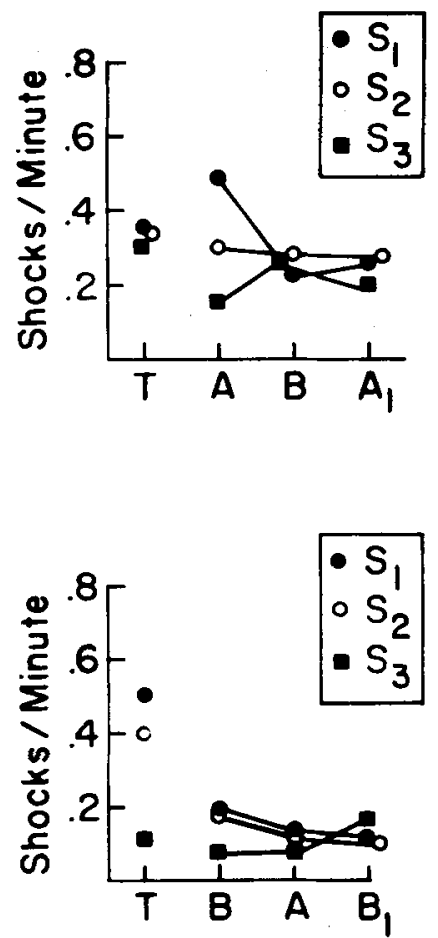

Fig. 2. Shock rates for the last training session ( $T$ ), and mean shocks per minute over four sessions over successive 30-min periods. A, feedback condition; B, feedback removed.

proprioceptive-result in a generalization decrement. Accordingly, the transition from Condition $T$ to $B$, and from $A$ to $B$, would be expected to result in a decline of response rates. Neither prediction was supported by the results of this experiment since neither condition resulted in a decline of responding; on the contrary, Condition $\mathrm{BAB}_{1}$ led to an increase of response rate, while response rate under Condition $\mathrm{ABA}_{1}$ was maintained.

The following analysis is presented in explanation of our results. In an FOA situation, the response-produced stimulus signals a "safety" period during and following which a $S$ tends not to respond (Anger, 1963; Rescorla, 1968) so that the total response rate is reduced. An FS, therefore; acts as a stimulus for "response pacing." Under Condition $\mathrm{BAB}_{1}$, where the pacing stimulus is absent at the start, FOA performance may deteriorate, resulting in an increase of shock and leading the $S$ to increase his response rate. The subsequent high rate of response as a consequence of being shocked (Sidman, 1958) would be expected to maintain itself since it leads to avoidance. During the period of $\mathrm{ABA}_{1}$, the $S$ seems to maintain the rate of response developed under $\mathbf{A}$, and would probably only change this rate if major rather than supportive stimulus changes occurred during the session. By implication, we regard the enhanced feedback stimulus as supportive of a response rate, rather than its primary determinant. This function must be attributed to the parameters of the avoidance schedule.

In general, the results of this experiment support the proposition that, with extensive training, a $S$ relies less and less upon external and proprioceptive stimuli in the maintenance of a conditioned rate of response. Changes in response feedback made in the early part of a session, at a time when the pattern of responding for that session is developing, i.e., during the "warm-up" period, may affect the rate for the remainder of the session, provided it does not interfere with avoidance. In short, across-session behavior (as from $\mathrm{T}$ to $\mathrm{B}$ ) rather than within-session behavior (as from $A$ to B) seems to be effected by manipulations of the feedback stimulus.

\section{REFERENCES}

ADAMS, J. A. Response feedback and learning. Psychological Bulletin, 1968, 70, 486-504.

ANGER, D. The role of temporal discrimination in the reinforcement of Sidman avoidance behavior. Journal of the Experimental Analysis of Behavior Supplement, 1963,6, 477-506.

BOLLES, R. C., \& GROSSEN, N. E. Effects of an informational stimulus on the acquisition of avoidance behavior in rats. Joumal of Comparative \& Physiological Psychology, 1969, 68,90-99.

BOLLES, R. C., \& POPP, R. J. Parameters affecting the acquisition of Sidman avoidance. Journal of the Experimental Analysis of Behavior, 1964, 7, 315-321.

BOLLES, R.C., \& WARREN, J. A. The acquisition of bar-press avoidance as a function of shock intensity. Psychonomic Science, 1965, 3, 397-398.

LEAF, R. C. Acquisition of Sidman avoidance responding as a function of S-S interval. Journal of Comparative \& Physiological Psychology, $1965,59,298-300$.

LEAF, $R$. C. Some effects of response consequences on Sidman avoidance acquisition Journal of Comparative \& Physiological Psychology, 1966, 61, 217-220.

RESCORLA, R. A. Pavlovian conditioned fear in Sidman avoidance learning. Journal of Comparative \& Physiological Psychology, 1968, 65, 55-60.

SANDLER, J., DAVIDSON, R. A., \& HOLZSCHUH, R. D. Effects of increasing punishment intensity on Sidman avoidance behavior. Psychonomic Science, 1966, 5 , 103-104.

SIDMAN, M. Some notes on "bursts" in free-operant avoidance experiments. Journal of the Experimental Analysis of Behavior, 1958, 1, 167-172.

\section{NOTE}

1. This research was supported by NSF Grant GB-8505.

\section{Some effects of scopolamine on locomotor activity in rats}

BARTON MEYERS and ROBERT $C$. WILCHIN, Brooklyn College of the City University of New York, Brooklyn, N.Y. 11210

On 2 consecutive days, rats $(N=30)$ were injected subcutaneously with saline, $0.2 \mathrm{mg} / \mathrm{kg}$ methscopolamine, or $0.2 \mathrm{mg} / \mathrm{kg}$ scopolamine, and tested for their levels of locomotor activity. Scopolamine produced hyperactivity on both days, while methscopolamine induced no effect different from that of saline. These results were discussed in relation to the suggestion of Calhoun \& Smith (1968) that scopolamine impairs passive avoidance due to its effect on activity.

The systemic circulation of muscarinic cholinergic blocking drugs, such as atropine and scopolamine, increases the locomotor activity of both mice (Harris, 1961; Tripod, 1957) and rats(Meyers, Roberts, Riciputi, \& Domino, 1964; Payne \& Anderson, 1967; Pradham \& Roth, 1968; Tapp, 1965). Similarly, these agents, if injected at a time calculated to produce peak effects during training, impair passive avoidance behaviors in both mice (Bohdanecký \& Jarvik, 1967; Calhoun \& Smith, 1968; Stark, 1967) and rats (Bignami \& Gatti, 1968; Burešová, Bureš, Bohdanecký, \& Weiss, 1964; Meyers, 1965; Meyers \& Lazarus, 1967). Conversely, if the peak effect occurs shortly after the trial, i.e., during the presumed consolidation period, no passive-avoidance deficit results (Bohdanecký \& Jarvik, 1967; Burešová et al, 1964; Calhoun \& Smith, 1968).

Data such as these have led Calhoun \& Smith (1968) to conclude that anticholinergic agents exert their effect on the performance of passive avoidance, rather than on the learning of it. Specifically, they have suggested that scopolamine makes Ss "more excitable" or "more active" and that this condition impairs their ability to withhold the appropriate response in the passive-avoidance situation.

However, it has been demonstrated that the passive-avoidance behavior of rats can become impervious to repeated administrations of scopolamine (Bignami \& Gatti, 1968; Meyers, 1965; Meyers \& Lazarus, 1967). If successive scopolamine injections should continue to increase locomotor activity, though, it would be 